\title{
Prevalence and molecular distribution of Legionella pneumophila in cold water taps across Alborz province, Iran
}

\author{
Somayeh Yaslianifard ${ }^{\mathrm{a}, \mathrm{b}}$, Mohammad Mohammadzadeh ${ }^{\mathrm{a}}$, Mohammad Reza Pourmand ${ }^{\mathrm{c}}$, \\ Sahar Yaslianifard ${ }^{\mathrm{d}}$, Mohammad Noori Sepehr ${ }^{\mathrm{e}}$, Maryam Arfaatabar ${ }^{\mathrm{c}}$, Mehdi Norouzi ${ }^{\mathrm{f}, *}$ \\ ${ }^{a}$ Department of Microbiology, School of Medicine, Alborz University of Medical Sciences, Karaj, Iran \\ ${ }^{\mathrm{b}}$ Dietary Supplements and Probiotic Research Center, Alborz University of Medical Sciences, Karaj, Iran \\ ${ }^{\mathrm{c}}$ Department of Pathobiology, School of Public Health, Tehran University of Medical Sciences, Tehran, Iran \\ ${ }^{\mathrm{d}}$ Department of Bacteriology, Faculty of Medical Sciences, Damghan Branch, Islamic Azad University, Damghan, Iran \\ ${ }^{\mathrm{e}}$ Department of Environmental Health Engineering, Research Center for Health, Safety and Environment (RCHSE), Alborz University of Medical Sciences, Karaj, Iran \\ ${ }^{\mathrm{f}}$ Department of Virology, School of Public Health, Tehran University of Medical Sciences, Tehran, Iran
}

\section{A R T I C L E I N F O}

\section{Keywords:}

L. pneumophila

P. aeruginosa

Acinetobacter spp

Antibiotic resistance

Phylogenetic analysis

\begin{abstract}
A B S T R A C T
Background: The aim of this study is to explore the distribution of Legionella pneumophila (serogroups 1-6) in cold water taps across Alborz province, Karaj, Iran. In addition, some other non-fastidious gram-negative bacteria not belonging to the Enterobacteriaceae family (GNB-NE) were followed.

Subject: A total of 162 water samples were collected from 6 countries and tested by culture and genus-specific polymerase chain reaction (PCR). The L. pneumophila isolates were subsequently analyzed by monoclonal antibodies (MAbs) for serogroup identification, molecularly by PCR, and sequencing for genetic diversity. The patterns of resistance to antibiotics were determined by disk diffusion.

Results: Fifteen (9.25\%) samples were detected as positive for L. pneumophila by PCR and 12 (7.4\%) samples yielded growth in culture. All isolates of $L$. pneumophila were belonged to serogroup 1 . Fifteen GNB-NE species were isolated from the samples, including nine isolates of Pseudomonas aeruginosa and six isolates of Acinetobacter spp. Antibiotic resistance was detected in 3 isolates of $P$. aeruginosa.

Conclusions: Despite the prevalence of L. pneumophila, few clinical cases have been reported, which may be due to underdiagnosis of the disease caused by it. Therefore, the prompt attention should be paid to do clinical diagnosis of legionellosis by the effective diagnostic tests from patients with compatible clinical symptoms.
\end{abstract}

\section{Introduction}

Legionella pneumophila (L. pneumophila) bacteria was discovered following a pneumonia outbreak at the 1976 American Legion Convention in Philadelphia. ${ }^{1}$ It was isolated from the infected lung tissue and caused the epidemic Legionnaires' disease. ${ }^{2}$ It is known that pneumonia comes to pass in approximately $95 \%$ of the $L$. pneumophila infections. ${ }^{3}$ It rarely causes Pontiac fever, which is an influenza-like infection in humans. ${ }^{4}$

Until now, 53 species of the Legionella genus has been known, of which seven of them are divided into serogroups. ${ }^{5,6}$ The bacterial strains in these serogroups are genetically homologous (based on DNA hybridization experiments); however, they are discernible through their specific reactivity to the antibodies. ${ }^{1}$ Since now, the relationship between the infection by eighteen species of $L$. pneumophila and patients with pneumonia has been found. ${ }^{5}$

The incidence of legionellosis has been reported worldwide ${ }^{7}$; however, the accurate occurrence is usually undetermined due to the improper surveillance of the infected cases. The early detection of legionellosis relies on the increase of the physician's awareness about disease and development the requirement equipment to diagnose it. ${ }^{7,8}$ The most preferable place for $L$. pneumophila is the aquatic environment. ${ }^{8}$ According to the published reports, L. pneumophila can grow in biofilms, since it has adequate interplay with other organisms. It causes the increase of the survival duration and proliferation in water. ${ }^{9-11}$ Therefore, they are resistant to the standard water disinfection methods and can pollute the drinkable water supplies. ${ }^{9,11}$ Moreover, studies revealed the widespread incidence of $L$. pneumophila in the freshwater resources and marine waters. ${ }^{12,13}$

Although some phenotypic characteristics including gram stain, cell

\footnotetext{
* Corresponding author.

E-mail address: mnorouzi@tums.ac.ir (M. Norouzi).
} 
membrane fatty acid and ubiquinone content, morphology, and growth on the specific media are usually employed to diagnosis the L. pneumophila bacteria, more specific diagnostic techniques comprising DNA analysis and antigenic analysis should be utilized to discern individual species. ${ }^{2,5,14}$

For the first time, this study aimed to investigate the prevalence and genetic diversity of $L$. pneumophila in the cold water taps of the Alborz province. In addition, some other non-fastidious gram-negative bacteria not belonging to the Enterobacteriaceae family (GNB-NE) were traced and their antibiotic resistance were investigated.

\section{Methods}

\subsection{Sample collection}

Alborz Province is situated in North-West of Tehran. It has six counties, Karaj, Savojbolagh, Taleqan, Eshtehard, Fardis, and Nazarabad. A total of 162 samples were collected between 2015 and 2016, from 24 sampling sites along 6 counties. $1000 \mathrm{ml}$ of water taps were filled into sterile non-toxic $1000 \mathrm{~mL}$ plastic Bottles and then the samples were transferred to the microbiology lab in less than $4 \mathrm{~h}$.

\subsection{Sample preparation and DNA extraction}

The filtered samples were used for DNA extraction. The samples were filtered using cellulose nitrate membrane filters with a pore size of $0.45 \mu \mathrm{m}$ (Sartorius AG, Goettingen, Germany). Membranes were broken into small pieces inside the $30 \mathrm{ml}$ sterile plastic container with $25 \mathrm{ml}$ original filtered water. The container was shaken at $37^{\circ} \mathrm{C}$ for $24 \mathrm{~h}$ in order to release the bacteria from filter to the filtered water. Then, $10 \mathrm{ml}$ subsample was taken from each filtered samples and treated by heating at $50{ }^{\circ} \mathrm{C}$ for $30 \mathrm{~min}$ to inactivate the organisms except L. pneumophila spp. ${ }^{15}$ After treatment, $100 \mu \mathrm{l}$ of each water sample was inoculated on blood agar and BCYE (Becton, Dickinson and Company, MD, USA) medium supplemented with glycine, vancomycin, cyclohexamide, and polymyxin B (GVPC).The plates were incubated under microaerophillic conditions at $35^{\circ} \mathrm{C}(90 \%$ humidity, $3 \%$ of CO2) for 7 days. ${ }^{15}$

The gram negative rods or coccobacilli were recognized as $L$. pneumophila if they grow on BCYE agar and showed positivity in catalase and oxidase tests. ${ }^{16}$ Direct fluorescent antibody kit (Prolab, Texas, USA) was used to determine the serogroups.

To recover GNB-NE, $10 \mathrm{ml}$ of each filtered samples were placed on the tryptic soya agar medium. ${ }^{17}$

\subsection{Polymerase chain reaction (PCR) assay}

DNA was extracted using the PrimePrep Genomic DNA isolation kit (GENET-BIO). For molecular identification of $L$. pneumophila, following primers of mip gene were used in PCR test. F: GGCATAGATGTTTATC CGG, R: GTGAAACCTGAAAACTTGCT. The mip gene of L. pneumophila encodes a $24 \mathrm{kDa}$ protein that promotes the entry of bacteria into macrophages and amoeba. ${ }^{18,19}$ The final optimized PCR reaction consisted of $1 \mu \mathrm{l}$ of each primer ( $10 \mathrm{pmol}), 0.5 \mu \mathrm{lNTP}(10 \mathrm{mM}), 0.5 \mu \mathrm{l}$ $\mathrm{MgCl}(100 \mathrm{mM}), 0.2 \mu \mathrm{l}$ (1 unit) Taq DNA polymerase (Metabion, Germany), $2.5 \mu \mathrm{l}$ PCR buffer (10X), and $0.5 \mu \mathrm{l}$ of DNA template $(100 \mu \mathrm{g} / \mathrm{ml})$ in total volume of $25 \mu \mathrm{l}$ with double distilled water. The cycling program was adjusted as follows: 35 cycles of $1 \mathrm{~min}$ at $94^{\circ} \mathrm{C}, 30 \mathrm{~s}$ at $49^{\circ} \mathrm{C}$, and $30 \mathrm{~s}$ at $72{ }^{\circ} \mathrm{C}$, followed by a final extension of $6 \mathrm{~min}$ at $72{ }^{\circ} \mathrm{C}$.

\subsection{DNA sequencing and phylogenetic analysis}

Firstly, the PCR products were purified using Sephadex. ABI (Applied Biosystems) BigDye 3.1 Chemistry was used for cycle sequencing. Double-stranded sequencing runs were performed on 3130 Genetic Analyzer (Applied Biosystems).The mip gene was used to identify the L. pneumophila genotypes. The sequencing outcomes were collected and aligned with each other using BioEdit software (http:// www.mbio.ncsu.edu/bioedit/bioedit.html). The genotyping was carried out by phylogenetic analysis with reference sequences. The reference sequences were obtained from GenBank database (NCBI). Phylogenetic tree reconstruction was performed using MEGA software (version 6.06) (http://www.megasoftware.net), with the Maximum likelihood method (ML) in Kimura-two parameter substitution model with 1000 bootstrapping replicates.

\subsection{Isolation and identification of GNB-NE}

The tryptic soya agar (Merck KGaA, Darmstadt, Germany) and Pseudomonas selective agar base (Cetrimide Agar, Merck, Germany) were used for isolation of GNB-NE. The inoculated tryptic soya agar plates were incubated for $24 \mathrm{~h}$ at $37^{\circ} \mathrm{C}$. The grown colonies were counted and differentiated by biochemical test. The plates containing Pseudomonas selective agar base were incubated at $42^{\circ} \mathrm{C}$, and then positive oxidase test was performed for confirmation of the colonies. ${ }^{15,17}$

\subsection{Antimicrobial susceptibility tests}

Antimicrobial susceptibility tests were performed using disk diffusion method on Mueller-Hinton agar. Antibiotics used in the disk diffusion method were chosen randomly from different antibiotic classes, which were including cephalothin $(30 \mu \mathrm{g})$, cefepime $(30 \mu \mathrm{g})$, ceftazidime $(30 \mu \mathrm{g})$, ciprofloxacin $(5 \mu \mathrm{g})$, meropenem $(10 \mu \mathrm{g})$, imipenem $(10 \mu \mathrm{g})$, piperacillin/tazobactam $(10 / 100 \mu \mathrm{g})$, gentamicin $(10 \mu \mathrm{g})$, colistin, gatifloxacin $(5 \mu \mathrm{g})$, ampicillin $(10 \mu \mathrm{g})$, and amikacin $(30 \mu \mathrm{g})$. All antibiotic disks were purchased from Mast Company (UK).

\subsection{Study selection \& data extraction}

In order to perform a comparative study, some related reports regarding to prevalence of L. pneumophila in Iran published between 2010 and 2018 were found in MEDLINE (PubMed) and the Institute for Scientific Information (ISI) database. Then, they were screened at three levels: title, abstract and main text levels. A total of 13 eligible papers were selected for further consideration. Afterwards, the following information was extracted from each study: the first author, year of survey, region, sample, sample size, confirmed cases, and prevalence.

\section{Results}

\subsection{Isolation and identification of L. pneumophila strains}

From 162 water samples, 15 (9.25\%) were positive for L. pneumophila by PCR and $12(7.4 \%)$ yielded growth in culture, which indicates a higher sensitivity of PCR in comparison with culture method. The number of CFUs for L. pneumophila in positive culture was $100 \mathrm{CFU} /$ $100 \mathrm{ml}$. We also isolated 6 isolates of Legionella other than pneumophila that grew on GVPC agar. The PCR were positive only for one sample for these isolates, because mip gene is solely present in the L. pneumophila genome. Nine isolates of $L$. pneumophila belonged to serogroup 1 as determined by direct fluorescent antibody. The remaining isolates were not identified as serogroup (Fig. 1).

\subsection{Genotyping and phylogenetic analysis}

To confirm genotyping by direct fluorescent antibody, a 402 bp PCR product of the macrophage infectivity potentiator protein (mip) was used to perform the phylogenetic analysis with the corresponding regions of 12 reference sequences of $L$. pneumophila serogroups 1-6, which are available in the GenBank database. The six samples that were not detected by serology tests in addition to one confirmed sample were 


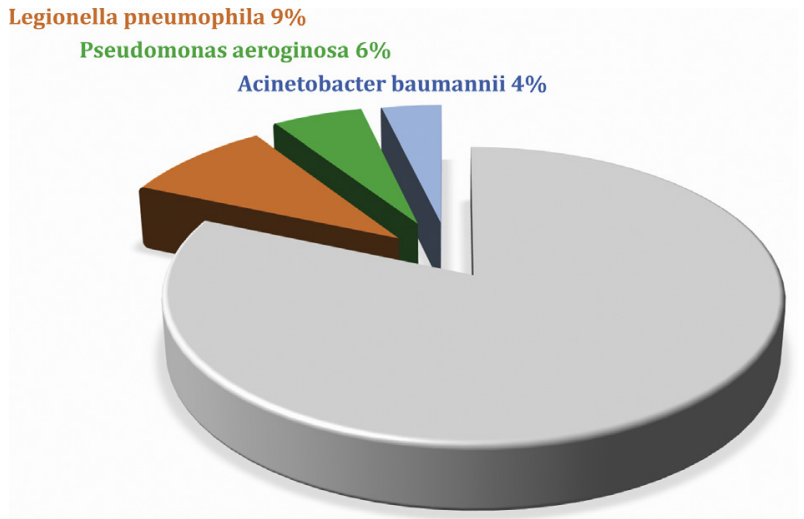

Fig. 1. Distribution of L. pneumophila, P. aeruginosa, and acinetobacter baumannii in samples of cold water tap in Alborz province, Iran.

sequenced to construct the phylogenetic tree. The nucleotide sequence analysis of seven isolates confirmed the circulating of $L$. pneumophila serogroup 1 in the Alborz province (Fig. 2). The L. pneumophila sequences demonstrated $98-99 \%$ homology with serogroup 1 sequences available in the GenBank database.

\subsection{Isolation of (GNB-E) and GNB-NE in water samples}

Gram-negative bacteria belonging to the Enterobacteriaceae family (GNB-E) were not found in the examined water samples. Fifteen GNBNE species were isolated from the water samples, which included nine isolates of $P$. aeruginosa and six isolates of Acinetobacter spp. The number of CFUs detected for $P$. aeruginosa and Acinetobacter spp. were ranged from 50 to $300 \mathrm{CFU} / 100 \mathrm{ml}$ and $20-100 \mathrm{CFU} / 100 \mathrm{ml}$, respectively. Three samples were simultaneously positive for $L$. pneumophila

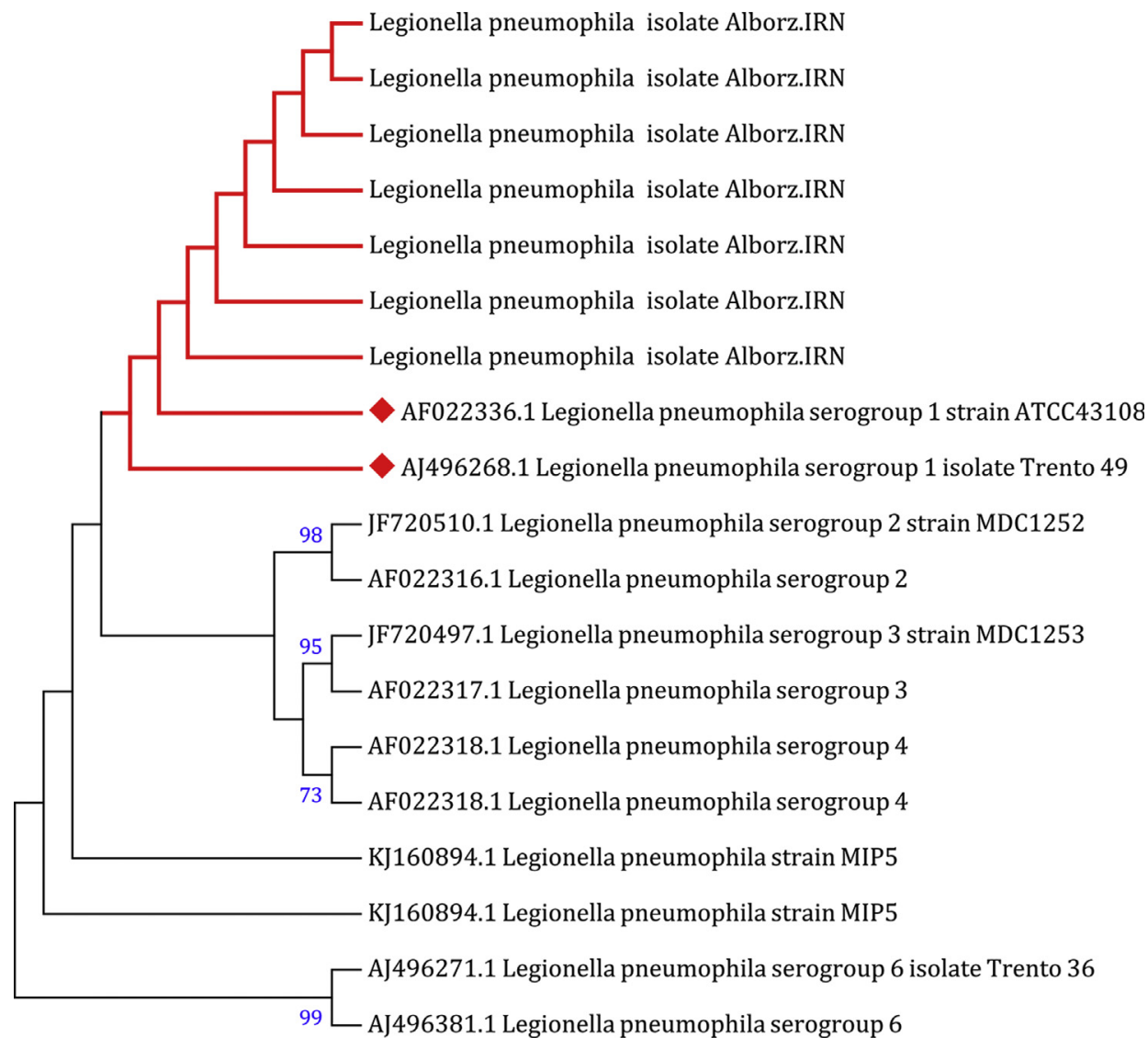

and P. aeruginosa, (Fig. 1).

\subsection{Antibacterial susceptibility testing}

In this study, only colistin antibiotic resistance was detected for 3 isolates of $P$. aeruginosa based on CLSI reference guide-lines for disk diffusion method.

\subsection{Study selection \& data extraction}

Table 1 summarizes different reports on distribution of L. pneumophila in water of different regions and provinces of Iran (Table 1). The highest prevalence was reported by Asghari. F. B, which was found in the hospital water systems of Isfahan in $2013 .{ }^{20}$ After that, the prevalence of $41.17 \%$ was reported by Tabatabaei. $M$. in the cold and warm water systems of South-West of Iran in $2016 .^{21}$

\section{Discussion}

The pollution of water resources with $L$. pneumophila species can exacerbate the incidence of Legionnaires' disease. Therefore, the public water resources should be investigated in terms of the presence of $L$. pneumophila.

This report disclosed the presence of 15 and $12 \mathrm{~L}$. pneumophila -positive samples using PCR and growth in culture techniques, respectively, in the cold water taps of Alborz province, Iran. However, only nine samples of 15 PCR-confirmed were detected by serology tests. The serogroup of nine isolates of $L$. pneumophila was determined as serogroup 1. In order to determine the serogroup of the other 6 PCRconfirmed, the phylogenetic tree was constructed and disclosed the same serogroup. In addition, the presence of six isolates of $L$. pneumophila was found.

Water is a proper medium for living L. pneumophila, which leads to

Fig. 2. The subtyping of L. pneumophila by mip gene sequences characterized in samples of cold water tap in Alborz province, Iran. Phylogenetic tree reconstruction was conducted using MEGA software (version 6.06), with the maximum likelihood method in the Kimura two parameter substitution model with 1000 bootstrap sampling. All obtained sequences made the same clade with the serogroup 1 . 
Table 1

Summary of the selected studies conducted on L. pneumophila distribution in water of different regions and provinces of Iran.

\begin{tabular}{|c|c|c|c|c|c|c|c|}
\hline Row & Region & Sample & Sample size & Confirmed cases & Detection method & Prevalence & Ref \\
\hline 1 & South-West of Iran & Cold and warm water systems & 34 & 14 & PCR & $41.17 \%$ & 21 \\
\hline 2 & Tehran & Water and dialysate samples & 50 & 4 & PCR & $8 \%$ & 22 \\
\hline 3 & Tehran & Hot and cold water systems & 150 & 33 & Biochemical tests & $22 \%$ & 23 \\
\hline 4 & Kerman and Bam & Cooling water systems & 128 & 25 & Real Time PCR & $19.50 \%$ & 24 \\
\hline 5 & Isfahan & Bio-aerosols generated from wastewater treatment plant & 54 & 3 & PCR & $6 \%$ & 25 \\
\hline 6 & Bandar-e-Abbas & Home air-conditioning system & 66 & 14 & Biochemical tests & $22 \%$ & 26 \\
\hline 7 & Tehran & Hospital Water Systems & 45 & 14 & PCR & $31.10 \%$ & 27 \\
\hline 8 & Tehran & Hospital water supplies & 45 & 13 & PCR & $29 \%$ & 28 \\
\hline 9 & Tabriz & Tap water of Tabriz hospitals & 140 & 10 & PCR & $14 \%$ & 29 \\
\hline 10 & Isfahan & Hospital water systems & 44 & 29 & PCR & $66 \%$ & 20 \\
\hline 11 & Tehran & ICU wards of Tehran hospitals & 52 & 5 & Real Time PCR & $9.60 \%$ & 30 \\
\hline 12 & Mashhad & Dental unit water line system & 52 & 19 & ELISA & 36.1 & 31 \\
\hline 13 & Khuzestan & Fishponds, swimming pools and cooling towers & 150 & 23 & PCR & 15.3 & 32 \\
\hline
\end{tabular}

its survival period in the human water resources and subsequently infectious possibility of consumers. ${ }^{33}$ The Legionnaires' disease caused by L. pneumophila infection has usually not been reported, because of inattention of clinicians to prescribe the laboratory tests and health authorities to trace the suspicious cases. ${ }^{34}$ Therefore, the detection of $L$. pneumophila in public community resources can lead to make serious decisions to prevent the prevalence of disease caused by it. ${ }^{35,36}$ The previously studies on the presence of $L$. pneumophila in Iran (Table 1) revealed high prevalence in different kind of water supply systems. Moreover, infection of hospitals water with $L$. pneumophila is more worrying, since the vulnerable groups are more exposed to the infection. ${ }^{20}$ Also, the water and wastewater treatment system of Iran should be more considered in terms of eliminating L. pneumophila from urban water systems.

The gold standard for detection of $L$. pneumophila is culture, which confronts with some limitations and problems including time-consuming, low sensitivity, rather than molecular techniques, negative grow after initiation of antibiotic therapy, and need to proper preprocessing of samples. PCR technique can resolve the mentioned obstacles and increase the detection accuracy. ${ }^{37,38}$ The selection of specific target regions of the $L$. pneumophila gene is important to reach precise results. To this end, we elected mip gene that covered all serogroups as specified by blasting of genetic databases. Using mip gene in PCR assay, 15 positive samples were detected among 162 water samples, while only 12 positive samples were found by culture. L. pneumophila serogroup 1 is the predominant cause of legionellosis in many, parts of the world, but in other areas, other serogroups are more important. For example, Legionella longbeachae is a major cause of legionellosis in Australia and New Zealand. ${ }^{39}$ On the other hand, serogroup 1 is more important in pathogenesis and induction of clinical signs. ${ }^{8}$ The genotyping results from this study confirmed the presence of serotype 1 in the water taps. This could have provide early warning to the relevant authorities.

Enterobacteriaceae is a large family of gram-negative, which usually housed in gastrointestinal tract of human and animals. Some members of this family have pathogenesis potential and are drug-resistant. In this study, the GNB-E was not detected in the water samples. However, nine isolates of Pseudomonas aeruginosa and six isolates of Acinetobacter spp. belonging to non-gram-negative Enterobacteriaceae were found. Moreover, the superinfection of $L$. pneumophila and $P$. aeruginosa were detected in three samples, which can lead to severe problems in the infected subjects. Although, only three isolates of $P$. aeruginosa showed colistin antibiotic resistance, the Enterobacteriaceae resistance must be considered in order to prohibit the following drug resistance among the infected cases.

\section{Conclusion}

The present study revealed that more attention should be paid to decontaminate of community water system from $L$. pneumophila, $P$. aeruginosa, and Acinetobacter spp. in Alborz Province, Iran. Moreover, the common serology technique cannot precisely detect the L. pneumophila; however, PCR seems to be a good replacement.

\section{Funding}

This project was financially supported by Alborz University of Medical Sciences, karaj, Iran.

\section{Conflicts of interest}

None declared.

\section{Acknowledgment}

The authors appreciate Dr. Sayed-Hamidreza Mozhgani as an expert for sequence management and phylogenetic analysis.

\section{References}

1. Brenner DJ. Classification of the legionellae. Semin Respir Infect. 1987;2:190-205.

2. Fang GD, Yu VL, Vickers RM. Disease due to the Legionellaceae (other than Legionella pneumophila). Historical, microbiological, clinical, and epidemiological review. Medicine. 1989;68:116-132.

3. Nguyen MH, Stout JE, Yu VL. Legionellosis. Infectious disease clinics of North America 1991;5:561-584

4. Hoge CW, Breiman RF. Advances in the epidemiology and control of Legionella infections. Epidemiol Rev. 1991;13:329-340.

5. Bangsborg JM. Antigenic and genetic characterization of Legionella proteins: contributions to taxonomy, diagnosis and pathogenesis. APMIS Suppl. 1997;70:1-53.

6. Duncan C, Prashar A, So J, et al. Lcl of Legionella pneumophila is an immunogenic GAG binding adhesin that promotes interactions with lung epithelial cells and plays a crucial role in biofilm formation. Infect Immun. 2011;79:2168-2181.

7. Hoge CW, Breiman RF. Advances in the epidemiology and control of Legionella infections. Epidemiol Rev. 1991;13:329-340.

8. Marston BJ, Lipman HB, Breiman RF. Surveillance for Legionnaires' disease. Risk factors for morbidity and mortality. Arch Intern Med. 1994;154:2417-2422.

9. Borella P, Guerrieri E, Marchesi I, Bondi M, Messi P. Water ecology of Legionella and protozoan: environmental and public health perspectives. Biotechnol Annu Rev. 2005;11:355-380.

10. Yu VL. Prevention and control of Legionella: an idea whose time has come. Infect Dis Clin Pract. 1997;6:420-421.

11. Lin YS, Stout JE, Yu VL, Vidic RD. Disinfection of water distribution systems for Legionella. Semin Respir Infect. 1998;13:147-159.

12. Ortiz-Roque CM, Hazen TC. Abundance and distribution of Legionellaceae in Puerto Rican waters. Appl Environ Microbiol. 1987;53:2231-2236.

13. Palmer CJ, Tsai YL, Paszko-Kolva C, Mayer C, Sangermano LR. Detection of Legionella species in sewage and ocean water by polymerase chain reaction, direct fluorescent-antibody, and plate culture methods. Appl Environ Microbiol. 1993;59:3618-3624.

14. Winn Jr WC. Legionnaires disease: historical perspective. Clin Microbiol Rev. 1988;1:60-81.

15. Veronesi L, Capobianco E, Affanni P. Legionella contamination in the water system of hospital dental settings. Acta Bio Medica Atenei Parmensis. 2007;78:117-122.

16. Blyth CC, Adams DN, Chen SC. Diagnostic and typing methods for investigating Legionella infection. New South Wales public health bulletin. 2009;20:157-161.

17. Stojek NM, Szymanska J, Dutkiewicz J. Gram-negative bacteria in water distribution 
systems of hospitals. Ann Agric Environ Med. 2008;15:135-142.

18. Wilson DA, Yen-Lieberman B, Reischl U, Gordon SM, Procop GW. Detection of Legionella pneumophila by real-time PCR for the mip gene. J Clin Microbiol. 2003;41:3327-3330.

19. Nomanpour B, Ghodousi A, Babaei T, Jafari S, Feizabadi M. Single tube real time PCR for detection of Streptococcus pneumoniae, Mycoplasma pneumoniae, Chlamydophila pneumoniae and Legionella pneumophila from clinical samples of CAP. Acta Microbiol Immunol Hung. 2012;59:171-184.

20. Asghari FB, Nikaeen M, Hatamzadeh M, Hassanzadeh A. Surveillance of Legionell species in hospital water systems: the significance of detection method for environmental surveillance data. J Water Health. 2013;11:713-719.

21. Tabatabaei M, Hemati Z, Moezzi MO, Azimzadeh N. Isolation and identification of Legionella spp. from different aquatic sources in south-west of Iran by molecular \& culture methods. Mol Biol Res Commun. 2016;5:215-223.

22. Nazemi S, Mirzaii M, Yaslianifard S, et al. Microbiological qualification of air, water and dialysate in a haemodialysis centre: a new focus on Legionella spp. Iran. J Microbiol. 2016;8:219-225.

23. Ghanizadeh G, Mirmohamadlou A, Esmaeli D. Predictive parameters of Legionella pneumophila occurrence in hospital water: HPCs and plumbing system installation age. Environ Monit Assess. 2016;188:536.

24. Ahmadrajabi R, Shakibaie MR, Iranmanesh Z, Mollaei HR, Sobhanipoor MH. Prevalence of mip virulence gene and PCR-base sequence typing of Legionella pneumophila from cooling water systems of two cities in Iran. Virulence. 2016;7:602-609.

25. Mirzaee SA, Nikaeen M, Hajizadeh Y, Nabavi BF, Hassanzadeh A. Detection of Legionella spp. by a nested-PCR assay in air samples of a wastewater treatment plant and downwind distances in Isfahan. Adv Biomed Res. 2015;4:48.

26. Alipour V, Mahvi AH, Rezaei L. Quantitative and qualitative characteristics of condensate water of home air-conditioning system in Iran. Desalin Water Treat. 2015;53:1834-1839.

27. Rafiee M, Mesdaghinia A, Hajjaran H, Hajaghazadeh M, Miahipour A, Jahangiri-Rad M. The efficacy of residual chlorine content on the control of Legionella spp. In hospital water systems. Iran J Public Health. 2014;43:637-644.

28. Rafiee M, Jahangiri-Rad M, Hajjaran H, Mesdaghinia A, Hajaghazadeh M. Detection and identification of Legionella species in hospital water supplies through
Polymerase Chain Reaction (16S rRNA). J Environ Health Sci Eng. 2014;12:83.

29. Ghotaslou R, Yeganeh Sefidan F, Akhi MT, Soroush MH, Hejazi MS. Detection of legionella contamination in tabriz hospitals by PCR assay. Adv Pharmaceut Bull 2013;3:131-134

30. Yaslianifard S, Mobarez AM, Fatolahzadeh B, Feizabadi MM. Colonization of hospital water systems by Legionella pneumophila, Pseudomonas aeroginosa, and Acinetobacter in ICU wards of Tehran hospitals. Indian J Pathol Microbiol. 2012;55:352-356.

31. Ajami B, Ghazvini K, Movahhed T, Ariaee N, Shakeri M, Makarem S. Contamination of a dental unit water line system by legionella pneumophila in the mashhad school of dentistry in 2009. Iran Red Crescent Med J. 2012;14:376-378.

32. Moosavian M, Dashti A. Isolation and identification of legionellosis agents from fishponds, swimming pools and cooling towers in Khuzestan province, Iran. Jundishapur J Microbiol. 2011;4:209-215.

33. Mulla SA, Revdiwala S. Assessment of biofilm formation in device-associated clinical bacterial isolates in a tertiary level hospital. Indian J Pathol Microbiol. 2011;54:561-564.

34. Fields BS, Benson RF, Besser RE. Legionella and Legionnaires' disease: 25 years of investigation. Clin Microbiol Rev. 2002;15:506-526.

35. Welti M, Jaton K, Altwegg M, Sahli R, Wenger A, Bille J. Development of a multiplex real-time quantitative PCR assay to detect Chlamydia pneumoniae, Legionella pneumophila and Mycoplasma pneumoniae in respiratory tract secretions. Diagn Microbiol Infect Dis. 2003;45:85-95.

36. Cloud JL, Carroll KC, Pixton P, Erali M, Hillyard DR. Detection of Legionella species in respiratory specimens using PCR with sequencing confirmation. J Clin Microbiol. 2000;38:1709-1712.

37. Goh KT, Ng DL, Yap J, Ma S, Ooi EE. Surveillance, prevention, and control of legionellosis in a tropical city-state. Am J Infect Contr. 2005;33:286-291.

38. Lund V, Fonahn W, Pettersen JE, Caugant DA, Ask E, Nysaeter A. Detection of Legionella by cultivation and quantitative real-time polymerase chain reaction in biological waste water treatment plants in Norway. $J$ Water Health 2014;12:543-554.

39. Murdoch DR. Diagnosis of Legionella infection. Clinical infectious diseases : an official publication of the Infectious Diseases Society of America; 2003:64-69. 